\title{
Sífilis gestacional na Cidade do Recife: uma análise dos casos no Distrito Sanitário
}

\author{
Gestational syphilis in the City of Recife: an analysis of cases in the health district \\ Sífilis gestacional en la Ciudad de Recife: un análisis de los casos en el Distrito de Salud
}

Recebido: 10/03/2021 | Revisado: 17/03/2021 | Aceito: 21/03/2021 | Publicado: 28/03/2021

\author{
Marcella Dantas Ribeiro \\ ORCID: https://orcid.org/0000-0001-6414-8205 \\ Secretaria da Saúde de Recife, Brasil \\ E-mail: marcelladantas73@gmail.com \\ Paolo Porciúncula Lamb \\ ORCID: https://orcid.org/0000-0002-6712-0168 \\ Secretaria da Saúde de Recife, Brasil \\ E-mail: paoloplamb@gmail.com \\ Mariana Nathália Gomes de Lima \\ ORCID: https://orcid.org/0000-0003-1218-5607 \\ Secretaria da Saúde de Recife, Brasil \\ E-mail: mariananglima@gmail.com \\ Andréa Loureiro Roges \\ ORCID: https://orcid.org/0000-0003-0109-2114 \\ Secretaria da Saúde de Recife, Brasil \\ E-mail: deiaroges@hotmail.com
}

\begin{abstract}
Resumo
Objetivo: O objetivo do presente estudo foi analisar os casos notificados de sífilis gestacional no Distrito Sanitário VII da cidade do Recife, Brasil. Método: Trata-se de um estudo documental, do tipo exploratório-descritivo, realizado através das fichas de notificação dos casos de sífilis gestacional, ocorridos no período entre janeiro de 2014 e dezembro de 2018, analisados a partir de estatística descritiva. Resultados: Foram identificados 247 casos durante o período de análise, com crescente aumento no ano de 2018. As mulheres tinham entre 20 e 24 anos de idade, tinham baixa escolaridade, eram pardas e tiveram diagnóstico no segundo e terceiro trimestre de gestação. Não houve diferenças significativas no perfil das gestantes ao longo dos anos. Conclusão: $\mathrm{O}$ crescente aumento dos casos mostrou uma fragilidade dos serviços de saúde durante o processo, demonstrando a necessidade do estímulo à notificação e preenchimento correto das fichas. Sendo assim, ressaltamos a importância da educação em saúde, com vistas a esclarecer os principais pontos relativos à doença, se utilizando de uma linguagem de fácil compreensão pela população.
\end{abstract}

Palavras-chave: Doenças Sexualmente Transmissíveis; Atenção integral à saúde da mulher; Treponema pallidum.

\begin{abstract}
Objective: To analyze there notified cases of gestational syphilis in the Health District VII, Recife, Brasil. Method: This is a documentary study, exploratory-descriptive, carried out through the notification forms of cases of gestational syphilis, which occurred in the period be tween January 2014 and December 2018, analyzed from descriptive statistics. Results: 247 cases were identified during the analysis period, with a higher in crease in 2018. The women were bet ween 20 and 24 year sold, had low education, were brown, and had a diagnosis in the second and third trimester of pregnancy. There were no significant differences in the profile of pregnant women over the years. Conclusion: The increasing number of cases showed a weakness in health services during the process, demonstrating the need to encourage notification and correct completion of the forms. There fore, we emphasize the importance of health education, in order to clarify the main points related to the disease, using a language that is easy for the population to understand.
\end{abstract}

Keywords: Sexually Transmitted Diseases; Comprehensive women's health care; Treponema pallidum.

\section{Resumen}

Objetivo: El objetivo del presente estudio fue analizar los casos notificados de sífilis gestacional en el Distrito Sanitario VII de la ciudad de Recife, Brasil. Método: Se trata de un estudio documental, exploratorio-descriptivo, realizado a través de los formularios de notificación de casos de sífilis gestacional, ocurridos en el período comprendido entre enero de 2014 y diciembre de 2018, analizados mediante estadística descriptiva. Resultados: se identificaron 247 casos durante el período de análisis, con un incremento creciente en el año 2018. Las mujeres tenían entre 20 y 24 años, tenían baja escolaridad, eran morenas y tenían un diagnóstico en el segundo y tercer trimestre del embarazo. No hubo diferencias significativas en el perfil de las embarazadas a lo largo de los años. Conclusión: El creciente número de casos mostró una debilidad en los servicios de salud durante el proceso, lo que demuestra la 
necesidad de incentivar la notificación y la correcta cumplimentación de los formularios. Por ello, destacamos la importancia de la educación en salud, con el fin de aclarar los principales puntos relacionados con la enfermedad, utilizando un lenguaje de fácil comprensión para la población.

Palabras clave: Enfermedades Sexualmente Transmisibles; Atención integral a la salud de la mujer; Treponema pallidum.

\section{Introdução}

A Sífilis é uma doença infecciosa crônica, que desafia há séculos a humanidade, causado por uma bactéria chamada Treponema Pallidum, sendo considerado um grande problema de saúde pública pela organização Mundial de Saúde (Saloojee et al, 2004).

Sua transmissão pode ocorrer por contato sexual na maioria dos casos, mas também pode ser por transfusão sanguínea de forma mais rara e também verticalmente através da placenta no período gestacional (Ministério da Saúde, 2019).

A apresentação do agravo em relação aos seus sinais e sintomas é muito variável e complexa, quando não tratada evolui para formas mais graves, podendo comprometer a pele, o sistema nervoso, o aparelho cardiovascular, o aparelho respiratório e o aparelho gastrointestinal. Em 1928, conseguiu-se controlar a sífilis, com a descoberta da penicilina por Alexander Fleming, porém as mudanças nos comportamentos sexuais da sociedade e o surgimento das pílulas anticoncepcionais nos anos 60 contribuíram para o ressurgimento da doença (Avalleira \& Bottino, 2006).

O seu diagnóstico é realizado por meios de testes laboratoriais e sua triagem realizada por um Teste Rápido Treponêmico (TR), com 20 minutos de tempo médio de resposta. Após a triagem, para confirmação da sífilis, faz-se necessário a realização de testes não treponêmicos, como Venereal Disease Laboratory (VDRL) ou Rapid Plasma Reagin (RPR), distinguindo a sorologia ativa de uma cicatriz sorológica (Ministério da Saúde, 2019).

Apesar do tratamento com penicilina ser considerado de baixo custo, ainda há o receio dos profissionais da Atenção Básica em executá-lo, devido a raras reações adversas, têm contribuído para perda do momento oportuno de tratamento (Ministério da Saúde, 2019).

Ainda que o diagnóstico e tratamento sejam de baixo custo e fácil acesso, e que seja uma patologia evitável, a sífilis representa uma grande preocupação, principalmente em mulheres em idade reprodutiva, devido ao risco da sífilis gestacional (SG), que compromete a formação fetal por poder ser transmitida verticalmente, ocasionando a sífilis congênita (Saloojee et al., 2004).

Para compreender melhor a extensão da doença e realizar intervenções necessárias ao controle, o Ministério da Saúde (MS), através da Portaria n³3, de 14 de julho de 2005, incluiu a SG na lista nacional de doenças de notificação compulsória (Brasil, 2005).

No Brasil, no período de 2005 a junho de 2019, no Sistema de Informação de Agravo de Notificação (SINAN), 324.321 casos de sífilis em gestantes foram notificados (Brasil, 2019), caracterizando assim uma nova epidemia de SG. Com isso, a presente pesquisa tem como objetivo analisar o perfil dos casos notificados de SG no Distrito Sanitário VII (DSVII) da cidade do Recife, nos anos de 2014 a 2018. Contribuindo assim, para ter uma melhor visualização do problema de saúde pública, além de oportunizar elaboração de intervenções de políticas públicas para uma situação de saúde mais adequada.

\section{Metodologia}

Trata-se de uma pesquisa documental, do tipo exploratório-descritiva. A pesquisa documental busca selecionar, tratar e interpretar a informação na sua forma bruta, com intuito de dar valor e sentido (Tavares, 2011). Quando a pesquisa se utiliza do estudo descritivo, há uma preocupação em informar o pesquisador sobre situações com exposição de características de determinada população a partir do uso de técnicas padronizadas de coleta de dados, descrevendo de forma real, sendo 
frequentemente utilizada em conjunto com as exploratórias, que são realizadas pelos pesquisadores preocupados com a atuação prática (Tavares, 2011).

A pesquisa foi realizada no Distrito Sanitário VII (DS VII), da cidade do Recife, no Setor de Vigilância Epidemiológica da Secretaria de Saúde do próprio DS. A amostra foi composta pelas fichas de notificação dos casos de sífilis gestacional, ocorridos no período de janeiro de 2014 a dezembro de 2018. Para analisar o número de casos de sífilis em gestantes e a variação da idade foram empregados uma análise de variância (ANOVA).

Para identificar a variação dos casos de sífilis diagnosticados em gestantes ao longo dos anos de acordo com a escolaridade, raça, idade gestacional, o esquema de tratamento da gestante, o esquema de tratamento do parceiro e motivo do não tratamento do parceiro foram utilizadas análises de covariância (ANCOVAs). Nos casos de diferenças significativas, utilizou o teste de Fisher post-hoc. Para associação entre o período gestacional em que o caso foi diagnosticado e o tipo de tratamento empregado utilizamos uma análise de Qui-quadrado. Na análise das relações entre as características das pacientes ao longo dos anos, foi utilizada uma análise de componentes principais (PCA). As variáveis foram padronizadas a partir do método de ranging, para possibilitar a comparação entre as diferentes características das gestantes.

O projeto de pesquisa foi analisado e aprovado pelo Comitê de Ética da Faculdade Pernambucana de Saúde, delegado pela Comissão Nacional de Ética em Pesquisa (CONEP) através da Plataforma Brasil, com número de protocolo 3.284.932 aprovado no dia 25 de Abril de 2019.

\section{Resultados e Discussão}

Foram encontrados 247 casos, sendo observadas diferenças significativas no número de sífilis em gestantes entre os anos de 2014-2018 ( $F 4,249=197,65 ; \mathrm{p}=0,0000$ ), que foram explicadas pelo aumento do número de casos em 2018, que diferiu significativamente de todos os anos anteriores. Ainda, sendo observadas diferenças sutis entre os anos de 2014, 2015, $2016 \mathrm{e}$ 2017 quando comparadas com o ano de 2018 (Tabela 1).

Tabela 1: Casos de sífilis em gestantes entre 2014-2018. Diferenças significativas (i.e., $p \leq 0,05$ ) entre os anos são destacadas em negrito.

\begin{tabular}{rrrrrrr}
\hline & 2014 & 2015 & 2016 & 2017 & 2018 \\
\cline { 2 - 7 } 2014 & & 0.123954 & 0.481434 & $\mathbf{0 . 0 0 5 9 6 7}$ & $\mathbf{0 . 0 0 0 0 1 7}$ \\
2015 & 0.123954 & & 0.797067 & 0.395603 & $\mathbf{0 . 0 0 0 0 1 7}$ \\
2016 & 0.481434 & 0.797067 & & $\mathbf{0 . 0 3 9 9 2 4}$ & $\mathbf{0 . 0 0 0 0 1 7}$ \\
2017 & $\mathbf{0 . 0 0 5 9 6 7}$ & 0.395603 & $\mathbf{0 . 0 3 9 9 2 4}$ & & $\mathbf{0 . 0 0 0 0 1 7}$ \\
2018 & $\mathbf{0 . 0 0 0 0 1 7}$ & $\mathbf{0 . 0 0 0 0 1 7}$ & $\mathbf{0 . 0 0 0 0 1 7}$ & $\mathbf{0 . 0 0 0 0 1 7}$ & \\
$\mathrm{F}_{4,249}=197,65 ; \mathrm{p}=0,0000$ & & & & \\
\hline
\end{tabular}

Fonte: Sinan, Vigilância Epidemiológica do Distrito Sanitário VII.

Não foram encontradas diferenças significativas na idade das gestantes diagnosticadas com sífilis ao longo dos anos ( $\mathrm{F} 4,241=1,4387 ; \mathrm{p}=0,22181$ ) sendo entre 20 e 24 anos a maior quantidade encontrada. Contudo, em 2014 a idade das pacientes variou bastante em relação aos anos que se sucederam. 
Mesmo que em diversos casos os campos escolaridade e raça tenham sido ignorados, o resultado evidenciou que os casos de sífilis em gestantes não diferiram em relação à escolaridade $(F 7,24=1,5283 ; p=0,20)$ ou em relação à raça declarada das pacientes ( $F 3,15=, 76500, p=, 53117)$, se mostrando um perfil de mulheres predominantemente com baixa escolaridade e pardas. Em relação ao tipo de tratamento o esquema terapêutico com 7.200 UI de Penicilína Benzatina foi o mais utilizado ao longo dos anos (Tabela 2).

Tabela 2: Fatores socioeconômicos. Valores significativos (i.e., $p \leq 0,05$ ) foram destacados em negrito.

\begin{tabular}{llll}
\hline Fatores & & $\mathrm{F}$ & $\mathrm{P}$ \\
\cline { 2 - 3 } Escolaridade & & 0,371858 & 0,5472 \\
& Ano & 2,5427 & 0,0636 \\
Raça & & 1,885474 & 0,0692 \\
& Ano & 3,877782 & 0,1784 \\
Idade Gestacional & & 2,95857 & 0,0716 \\
\multirow{2}{*}{ Tratamento -Gestante } & Ano & 16,51571 & $\mathbf{0 , 0 0 1 3}$ \\
\multirow{2}{*}{ Tratamento - Parceiro } & & 3,545217 & $\mathbf{0 , 0 0 8 4}$ \\
\multirow{2}{*}{ Motivo do não tratamento } & Ano & 9,016933 & $\mathbf{0 , 0 2 3 9}$ \\
& & 2,541630 & 0,0681 \\
& Ano & 22,24832 & $\mathbf{0 , 0 0 0 0}$ \\
& & 2,325300 & 0,0942 \\
& Ano & 13,76783 & $\mathbf{0 , 0 0 2 0}$ \\
\hline
\end{tabular}

Fonte: Sinan, Vigilância Epidemiológica do Distrito Sanitário VII.

Os casos de sífilis em gestantes, considerando a idade gestacional, não variaram ao longo dos anos, sendo nos segundos e terceiros trimestres de gestação a notificação dos casos.

O esquema de tratamento do parceiro e o motivo do não tratamento não diferiram ao longo dos anos (Figura 1). 
Figura 1: Dados sobre o tratamento aplicado nos parceiros de gestantes diagnosticadas com sífilis entre os anos de 2014-2018. A: Frequência de tratamentos ao longo dos anos; B: Frequência de casos indicando a motivação do não tratamento dos parceiros.
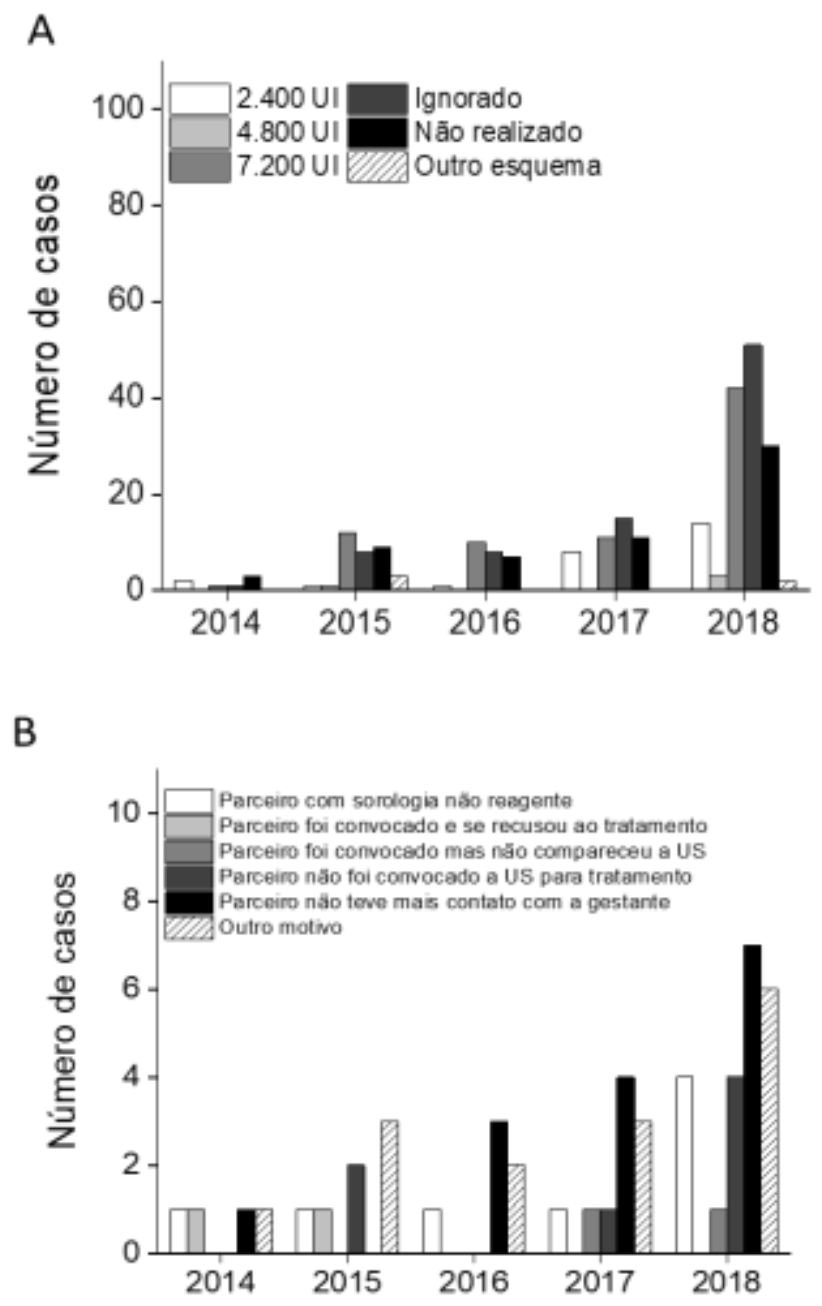

Fonte: Sinan, Vigilância Epidemiológica do Distrito Sanitário VII.

Não houve diferenças no perfil das gestantes em relação aos anos, apresentando vetores curtos e pouco relacionados com os dois principais eixos (Eixo 1 = 26,97 e Eixo 2 = 19,89) (Figura 2). 
Figura 2: Análise de componentes principais indicando as relações entre as características de gestantes diagnosticadas com sífilis entre os anos de 2014-2018.

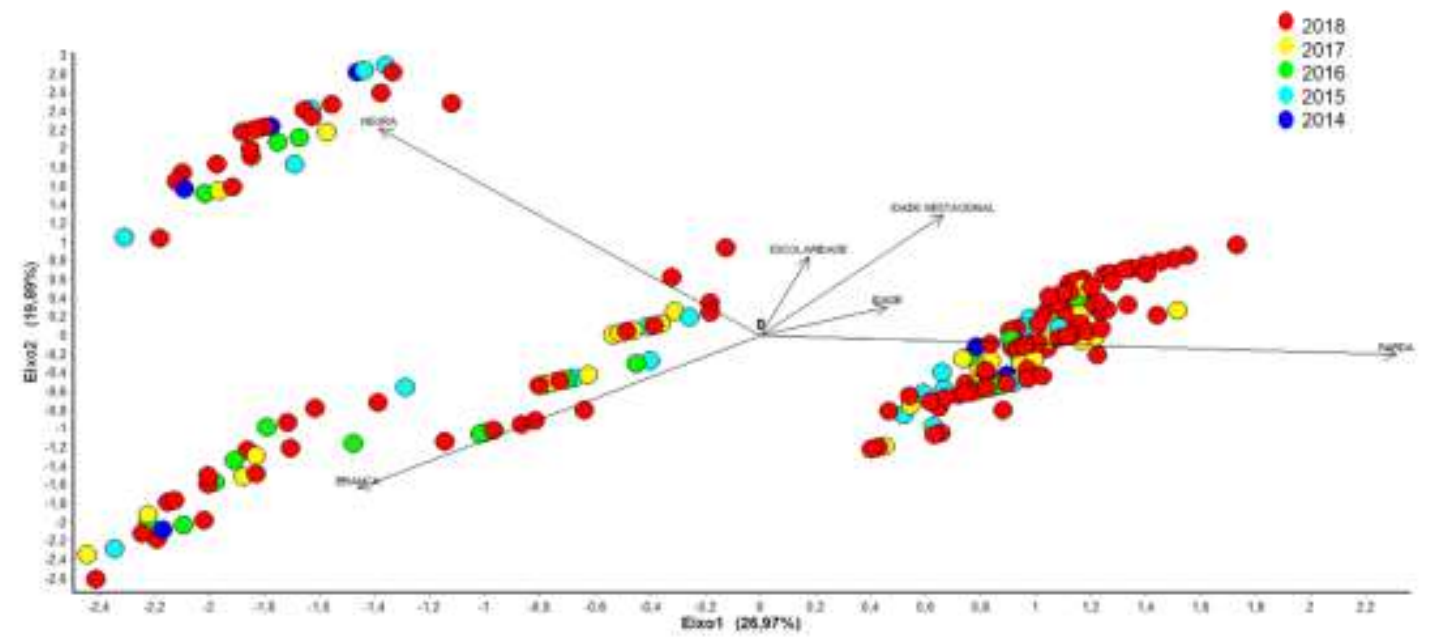

Fonte: Sinan, Vigilância Epidemiológica do Distrito Sanitário VII.

A pesquisa apresentou uma crescente significativa nos números das notificações, podendo estar relacionada com as mudanças nos critérios de definição da SG no final do ano de 2017, ao ser definido que toda mulher seria notificada com SG se o diagnóstico ocorresse entre os períodos de pré-natal, parto e/ou puerpério, como também com a ampliação da oferta dos testes rápidos (Avalleira \& Bottino, 2006). Outros fatores que podem estar associados às falhas no tratamento das gestantes e parcerias sexuais, ou ainda à redução da utilização de preservativos pela população (Maciel et al., 2017).

As faixas etárias com maiores índices foram encontradas entre 20 e 24 anos e se relacionam com a idade reprodutiva sexual das mulheres, estando diretamente ligado ao fator comportamental de risco, ao estar em contato com um maior número de parceiros e/ou serem sexualmente ativas, aumentando o risco de contato com o Treponema Pallidum (Oliveira, 2017).

Em relação ao critério raça/cor houve predominância de mulheres pardas, situação semelhante encontrada em outras regiões do país (Brasil, 2019).

Foi observado um baixo nível de escolaridade presente nas gestantes notificadas, fator que se associa a inúmeros fatores que limitam o processo saúde (Cardoso et al., 2018), além de limitação no entendimento das medidas de prevenção e fatores de risco (Serafim et al., 2014). Dados semelhantes relacionados a baixos níveis de escolaridade foram encontrados em outras regiões do país, mesmo sendo regiões que apresentam níveis de escolaridade mais altos que os da região nordeste (Padovani, Oliveira \& Pelloso, 2018).

No segundo e terceiro trimestre de gestação, foram identificados à maioria do diagnóstico dos casos, possivelmente relacionados à procura tardia de acompanhamento pré-natal e/ou a qualidade da assistência à gestante, fatores que comprometem a saúde materna infantil (Cavalcante, Pereira \& Castro, 2017) e se apresentam como uma barreira no controle da sífilis gestacional (Who, 2013).

Diante isso, percebe-se o baixo acompanhamento durante os primeiros meses de gestação destas mulheres na Atenção Básica, porém, de acordo com Cavalcante, Pereira \& Castro (2014) é nesse primeiro nível de atenção que se mostra estratégico no combate da sífilis congênita, devido à facilidade de acesso das equipes de Atenção Básica em conhecerem a história de vida das usuárias adscritas, dos vínculos entre profissional e paciente, que podem contribuir na mudança do quadro epidemiológico da sífilis congênita. 
Moreira Rodrigues et al. (2016) apontam que ações eficientes para prevenção e tratamento da sífilis pode ocorrer por meio de grupos de educação em saúde desenvolvidas na própria Atenção Básica, com gestantes, grupos de adolescentes em escolas e outros espaços da comunidade, a fim de atingir todas aquelas pessoas com risco de exposição a sífilis.

O tratamento da parceria sexual se apresenta como um fator de extrema importância para se evitar a reinfecção da gestante (Domingues et al., 2014), sendo recomendado que > 80,0\% das parcerias das gestantes diagnosticadas sejam tratados com, pelo menos, uma dose de Penicilina G Benzatina, mas os valores encontrados no estudo estão muito abaixo do recomendado, fato que compromete o tratamento adequado (Who, 2013).

\section{Considerações Finais}

Os resultados desse estudo demonstraram que ao longo dos anos houve um incremento nas notificações de SG, sendo na sua maioria mulheres entre 20 e 24 anos, pardas e com baixa escolaridade.

O diagnóstico na maioria foi realizado no segundo e terceiro trimestre de gestação o que evidencia que as gestantes estão recebendo assistência, mas não de maneira efetiva.

A utilização de dados secundários por vezes ocasiona a falta de informações necessárias para uma análise fidedigna do cenário que estão inseridas as gestantes, como foram notadas na variável de ocupação, que se apresentou ignorado em todas as fichas de notificação, impossibilitando a sua análise.

O não preenchimento adequado das fichas de notificação foi um fator limitante no processo de análise das informações, mesmo assim é possível visualizar que fatores sociais estão diretamente ligados à fragilidade dos serviços de saúde durante o processo, influenciando de maneira negativa no controle da sífilis gestacional, que se mantém durante anos.

Contudo, o perfil das notificações mostrou uma fragilidade dos serviços de saúde durante o processo, demonstrando ser necessário o estímulo à notificação e preenchimento correto das fichas para contribuir com as ações de monitoramento e controle, com ênfase na importância dos cuidados na assistência pré-natal, assim como, à prevenção da sífilis e o estímulo às notificações.

Ainda cabe apontar que a Atenção Básica é um campo privilegiado para detecção e tratamento da sífilis gestacional e a educação em saúde é uma estratégia potente, com vistas a esclarecer os principais pontos relativos à doença, e melhor adesão ao tratamento.

Diante do exposto, é importante ressaltar a relevância de trabalhos futuros sobre a temática em questão, no intuito de subsidiar a prática profissional na busca para redução e tratamento oportuno da sífilis gestacional.

\section{Referências}

Avelleira, J. C. R., \& Bottino, G. (2006). Sífilis: diagnóstico, tratamento e controle Syphilis: diagnosis, treatment and control. An bras dermatol, 81(2), 111-26. Brasil. (2019). Boletim Epidemiológico. Secretaria de Vigilância em Saúde.

Brasil. Ministério da Saúde (MS). (2005). Portaria $n^{\circ}$ 33, de 14 de Julho de 2005. Inclui doenças à relação de notificação compulsória, define agravos de notificação imediata ea relação dos resultados laboratoriais que devem ser notificados pelos Laboratórios de Referência Nacional ou Regional. Diário Oficial da União.

Cardoso, A. R. P., Araújo, M. A. L., Cavalcante, M. D. S., Frota, M. A., \& Melo, S. P. D. (2018). Análise dos casos de sífilis gestacional e congênita nos anos de 2008 a 2010 em Fortaleza, Ceará, Brasil. Ciência \& Saúde Coletiva, 23, 563-574.

Cavalcante, P. A. D. M., Pereira, R. B. D. L., \& Castro, J. G. D. (2017). Sífilis gestacional e congênita em Palmas, Tocantin s, 2007-2014. Epidemiologia e Serviços de Saúde, 26, 255-264.

Domingues, R. M. S. M., Szwarcwald, C. L., Souza Junior, P. R. B., \& Leal, M. D. C. (2014). Prevalência de sífilis na gestação e testagem pré-natal: Estudo Nascer no Brasil. Revista de Saúde Pública, 48, 766-774.

Maciel, R. B., de Barros, I. C., Ugrinovich, L. A., Simioni, P. U., \& de Oliveira, R. C. F. (2017). Epidemiologic profile of the cases of syphilis in AmericanaSP from 2005 to 2015. Journal of Epidemiology and Infection Control, 7(3), 161-168. 
Research, Society and Development, v. 10, n. 4, e1310413764, 2021

(CC BY 4.0) | ISSN 2525-3409 | DOI: http://dx.doi.org/10.33448/rsd-v10i4.13764

Ministério da Saúde (BR), Secretaria de Vigilância em Saúde, \& Departamento de Doenças de Condições Crônicas e Infecções Sexualmente Transmissíveis. (2019). Protocolo Clínico e Diretrizes Terapêuticas para Prevenção da Transmissão Vertical do HIV, Sífilis e Hepatites Virais.

Moreira Rodrigues, A. R., Monteiro da Silva, M. A., Sabino Cavalcante, A. E., Carvalho Araújo Moreira, A., Mourão Netto, J. J., \& Frota Goyanna, N. (2016). Atuação de enfermeiros no acompanhamento da sífilis na atenção primária. Journal of Nursing UFPE/Revista de Enfermagem UFPE, 10(4).

Oliveira, S. I. M. D. (2016). Notificações de sífilis gestacional e congênita: uma análise epidemiológica (Dissertação de Mestrado). Universidade Federal do Rio Grande do Norte, Natal, RN, Brasil.

Padovani, C., Oliveira, R. R. D., \& Pelloso, S. M. (2018). Sífilis na gestação: associação das características maternas e perinatais em região do sul do Brasil. Rev Latino-Am Enfermagem, 26, e3019.

Saloojee, H., Velaphi, S., Goga, Y., Afadapa, N., Steen, R., \& Lincetto, O. (2004). The prevention and management of congenital syphilis: an overview and recommendations. Bulletin of the World Health Organization, 82, 424-430.

Serafim, A. S., Moretti, G. P., Serafim, G. S., Niero, C. V., Rosa, M. I. D., Pires, M. M. D. S., \& Simões, P. W. T. D. A. (2014). Incidence of congenital syphilis in the South Region of Brazil. Revista da Sociedade Brasileira de Medicina Tropical, 47(2), 170-178.

Tavares, A. C., Sell, F., Sell, S., \& Unglaub, T. (2011). Metodologias para iniciação à prática da pesquisa e extensão. Florianópolis: UDESC/CEAD/UAB.

World Health Organization. (2012). Investment case for eliminating mother-to-child transmission of syphilis: promoting better maternal and child health and stronger health systems. Geneva: World Health Organization. 